\title{
Intraventricular Taenia solium Neurocysticercosis: A Report of Three Cases
}

Pant B, ${ }^{1}$ Devleesschauwer $B,{ }^{2}$ Shrestha $P,{ }^{1}$ Shrestha I, ${ }^{1}$ Praet $N,{ }^{3}$ Dorny $P^{2,3}$

'Annapurna Neurological Institute and Allied Sciences, Kathmandu, Nepal, ${ }^{2}$ Faculty of Veterinary Medicine, Ghent

University, Merelbeke, Belgium, ${ }^{3}$ Department of Biomedical Sciences, Institute of Tropical Medicine, Antwerp, Belgium

\section{ABSTRACT}

Neurocysticercosis (NCC), caused by the pork tapeworm Taenia solium, is reported to be a common condition in Nepal. So far imaging diagnosis was mainstay of the diagnosis. In this paper, we report three patients presenting with neurological symptoms due to intraventricular NCC. We have diagnosed the causative agent as T. solium on molecular basis. Further research is warranted to assess the actual health impact of $T$. solium in Nepal.

Keywords: Intraventricular neurocysticercosis, PCR-RFLP, Taenia solium

\section{INTRODUCTION}

Neurocysticercosis (NCC) is the infestation of the central nervous system with the larval stage or cysticercus of the pork tapeworm, Taenia solium. This infection occurs after the ingestion of tapeworm eggs, which are spread in the environment by human tapeworm carriers. ${ }^{1}$ Pork tapeworm is still endemic in various developing countries and regions, such as Nepal. Since several years, suspected NCC cases have been described in hospitals throughout this country, ${ }^{2,3}$ yet no study has been undertaken to confirm the causative agent. In this report, we present three patients with intraventricular NCC where molecular diagnosis was used to confirm $T$. solium as being the causative tapeworm species.

\section{CASE REPORTS}

\section{CASE 1}

A 70-year-old male patient presented with features of altered consciousness and disorientation. A brain CT scan showed communicating hydrocephalus with features of normal pressure hydrocephalus, without any clear sign of ventricular obstruction (Figure 1). The patient was treated by ventriculo-peritoneal shunting, which improved his condition significantly. After about four weeks, however, the patient again presented to the hospital with the same complaints. A new CT scan showed a recurrence of the hydrocephalus. Therefore, it was decided to repeat surgery by means of endoscopic third ventriculostomy (ETV). While performing ETV, a cystic lesion was identified in the ventricle and was endoscopically removed. However, the patient did not improve as the hydrocephalus did not resolve due to intraventricular inflammation, presumably caused by the toxic effect of the cyst fluid. The patient had to be put on a ventilator, and subsequently developed a fatal chest infection.

Correspondence:

Brecht Devleesschauwer

Faculty of Veterinary Medicine, Ghent University, Merelbeke, Belgium Salisburylaan 133,9820 Merelbeke, Belgium

Tel: +3292647328

Mail: brecht.devleesschauwer@ugent.be 


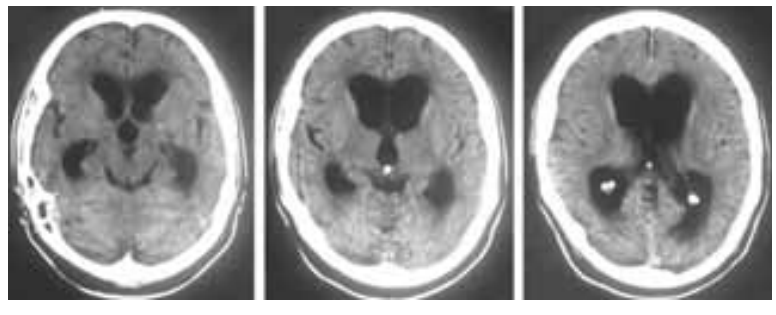

Figure 1. Sequential brain CT slices of Patient 1, showing communicating hydrocephalus without any obvious sign of obstruction within the ventricular system

\section{CASE 2}

An 18-year-old male patient presented with a twoday history of sudden headache associated with episodes of vomiting. There was no history of loss of consciousness, seizure or trauma. The patient appeared irritated and agitated, and showed gross papilloedema on fundoscopic examination. A brain CT scan showed communicating hydrocephalus, without any obvious indication of obstruction, as was the case in our first patient. The patient was treated by ETV, during which a cystic lesion occupying the lateral ventricle was indentified, which was endoscopically removed. At discharge, the patient's general condition was fair and his complaints of headache had disappeared.

\section{CASE 3}

A 48-year-old female patient presented with episodic headaches since the past 5 years. The patient had no history of loss of consciousness, vomiting or seizures. However, there was a history of nausea, blurring of vision and photophobia. The general condition of the patient appeared normal. A head CT showed features of obstructive hydrocephalus, while a brain MRI identified a cystic lesion in the right lateral ventricle. The cyst was surgically removed through ETV, and at discharge, the patient reportedly experienced no more headaches.

\section{Molecular diagnosis}

After endoscopic removal of the cystic lesions, they were preserved in $70 \%$ ethanol, and subjected to polymerase chain reaction followed by restriction fragment length polymorphism assay (PCR-RFLP), as described by Rodriguez-Hidalgo and colleagues. ${ }^{4}$ In brief, a PCR is first used to amplify two fragments of the mitochondrial $12 \mathrm{~S}$ ribosomal DNA of the sample, by using two specific primer pairs (i.e., ITMF/ITMTnR and TAEnF/ITMTnR). These fragments are then incubated with the restriction enzymes Ddel and $\mathrm{Hpy} 8 \mathrm{I}$, which will cut the amplified DNA at specific nucleotide sequences. By separating the thus obtained restriction fragments through polyacrylamide gel electrophoresis, a specific banding pattern is obtained that allows the distinction of the three Taenia species known to infect man, i.e., T. solium, Taenia saginata and Taenia asiatica. ${ }^{5}$ In all three patients, the PCR-RFLP analysis showed that the removed lesion was indeed a $T$. solium cysticercus (Figure 2).

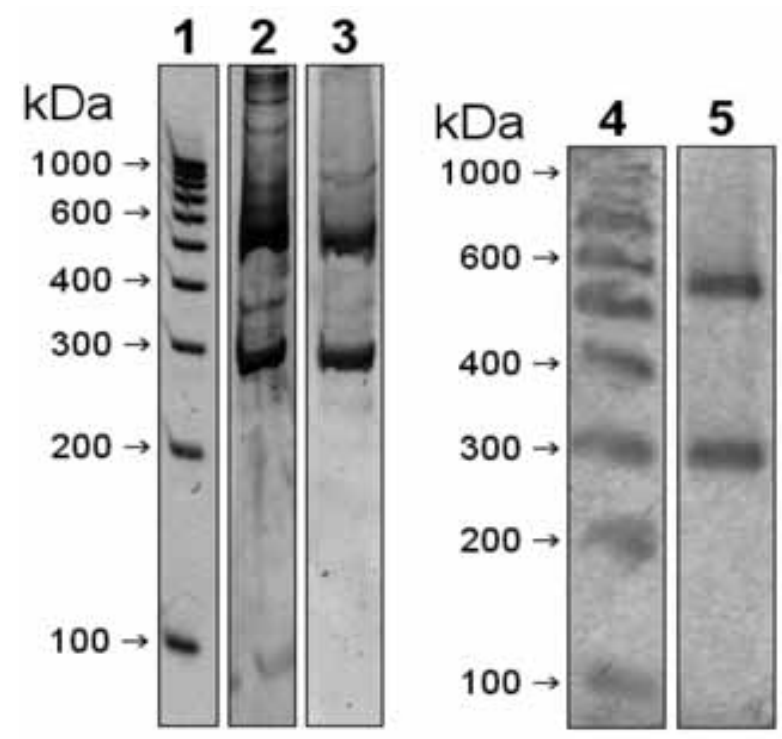

\section{DISCUSSION}

NCC is considered to be the most common parasitic infection of the central nervous system. Although being considered eradicable, it is still largely endemic in countries and regions where pigs are raised in primitive conditions and where pork is consumed raw or undercooked. ${ }^{1}$ In these endemic areas, NCC is a major cause of neurological disorders, most notably seizures and chronic headache, associated with parenchymal cysticerci. Intraventricular NCC is a less common appearance of NCC, occurring in less than 30 $\%$ of NCC patients, but is potentially more serious. ${ }^{6}$ Intraventricular cysticerci are mobile and can steadily increase in volume, thereby blocking the cerebrospinal fluid (CSF) flow, causing obstructive hydrocephalus. Persistent or intermittent symptoms of raised intracranial pressure are often the first clinical signs 
of this presentation, but sudden death has also been reported..$^{7}$ Therefore, immediate diagnosis and surgical intervention are imperative. The endoscopic excision of the cyst is currently the method of choice, since it is a minimally-invasive technique with good outcomes. ${ }^{8}$ In cases where hydrocephalus persists after cyst removal (e.g., due to ependymitis), ventriculo-peritoneal shunting is necessitated, although the outcomes are less favourable. ${ }^{8}$ In Case 1, ETV was not able to resolve the hydrocephalus, which indirectly led to the fatal outcome. Our second and third cases, on the other hand, recovered completely.

Although the appearance of hydrocephalus on CT or MRI is evident, determining its cause is less straightforward. Acquired hydrocephalus can result from obstructive intracranial masses, subarachnoid haemorrhage, head trauma, or infection. ${ }^{9}$ The most common acquired masses causing CSF blockage are choroid plexus papillomas and benign brain neoplasms, such as colloid cysts. In endemic areas, similar symptoms may be caused by cysticercotic and hydatid cysts, the latter being the larval stage of the small dog tapeworm Echinococcus granulosus. ${ }^{10}$ Differentiating these lesions on CT or MRI may not always be possible, and moreover, as in our first two patients, intraventricular cysticerci may even remain unnoticed with standard neuro-imaging techniques, since the density and intensity of the viable cysticerci are similar to those of the surrounding CSF. ${ }^{8}$ Therefore, histopathologic examination of the excised lesion is often performed to make the etiological diagnosis. However, morphology is not always conclusive to distinguish the different Taenia species. ${ }^{11}$ Indeed, even though $T$. solium is commonly cited as the causative agent of human NCC, there is still some controversy over the possibility of other Taenia species, most notably $T$. asiatica, to cause NCC in humans. ${ }^{12}$ Therefore, a definitive diagnosis of $T$. solium NCC can only be made by molecular analysis. ${ }^{11}$
Several molecular tools have been developed for making a species specific diagnosis of NCC..$^{4,5,13}$ In this study, we used a PCR complemented by RFLP to analyse the removed lesions, which in all three cases proved to be T. solium.

In conclusion, we confirmed using molecular diagnosis, to our knowledge for the first time, that $T$. solium is causing NCC in Nepalese patients. While the endemicity of this parasite in Nepal was first described over three decades ago, ${ }^{14}$ clinical studies on NCC have so far been limited to medical imaging studies, ${ }^{2,3}$ allowing only a presumptive diagnosis of $T$. solium NCC. Indeed, given the limited availability and high cost of molecular diagnosis in Nepal, the etiological diagnosis of NCC has so far remained impractical. In this study, molecular diagnosis was used to confirm the etiology of NCC, but we recognize that this currently cannot be recommended as a routine diagnostic tool for NCC in Nepal. In addition, as most reports are hospitalbased case studies, still little is known about the actual public health impact of the pork tapeworm in Nepal. Therefore, further research should focus on assessing the community impact of NCC, and on identifying the local risk factors for $T$. solium transmission. In term, this information will lead to a better understanding of the epidemiology of the pork tapeworm in Nepal, which will be invaluable for future control measures.

\section{ACKNOWLEDGEMENT}

The authors wish to thank Ms Inne Pauwels of the Institute of Tropical Medicine, Department of Animal Health, for performing the PCR-RFLP assays.

\section{REFERENCES}

1. Dorny P, Praet N, Deckers N, Gabriel S. Emerging foodborne parasites. Vet Parasitol. 2009;163:(3):196-206.

2. Piryani RM, Kohli SC, Shrestha G, Shukla A, Malla TB. Human neurocysticercosis managed at Nepalganj Medical College, Teaching Hospital, Kohalpur, Nepal. KUMJ. 2007;5;(4):518-20.

3. Shrestha BM. Childhood neurocysticercosis: clinicoradiological profile and outcome. J Nepal Paediatr Soc. 2008;28:(1):14-6.
4. Rodriguez-Hidalgo R, Geysen D, Benitez-Ortiz W, Geerts $\mathrm{S}$, Brandt J. Comparison of conventional techniques to differentiate between Taenia solium and Taenia saginata and an improved polymerase chain reaction-restriction fragment length polymorphism assay using a mitochondrial 12S rDNA fragment. J Parasitol. 2002;88:(5):1007-11.

5. Somers R, Dorny P, Geysen D, Nguyen LA, Thach DC, Vercruysse Jet al. Human tapeworms in north Vietnam. Trans R Soc Trop Med Hyg. 2007;101:275-7.

6. Goel RK, Ahmad FU, Vellimana AK, Suri A, Chandra PS, Kumar Ret al. Endoscopic management of intraventricular neurocysticercosis. J ClinNeurosci. 2008;15:1096-101. 
Pant et al. Intraventricular Taenia solium neurocysticercosis: a report of three cases

7. LlompartPou JA, Gené A, Ayestarán JI, Saus C. Neurocysticercosis presenting as sudden death. ActaNeurochir (Wien). 2005;147:785-6.

8. Rajshekhar V. Surgical management of neurocysticercosis. Int J Surg. 2010;8:100-4.

9. Hamilton MG. Treatment of hydrocephalus in adults. SeminPediatr Neurol. 2009;16:34-41.

10. Osborn AG, Preece MT. Intracranial cysts: radiologicpathologic correlation and imaging approach. Radiology. 2006;239:(3):650-64.

11. Ito A, Takayanagui OM, Sako Y, Sato MO, Odashima NS, Yamasaki Het al. Neurocysticercosis: clinical manifestation, neuroimaging, serology and molecular confirmation of histopathologic specimens. Southeast Asian J Trop Med Public Health. 2006;7:(3):74-81.
12. Galán-Puchades MT, Fuentes MV. Human cysticercosis and larval tropism of Taenia asiatica. Parasitol Today. 2000;16:(4):174.

13. Yamasaki H, Allan JC, Sato MO, Nakao M, Sako Y, Nakaya Ket al. DNA differential diagnosis of taeniasis and cysticercosis by multiplex PCR. J ClinMicrobiol. 2004;42:548-53.

14. Jimba M, Joshi DD. Health promotion approach for the control of food-borne zoonoses in Nepal: emphasis on an environmental assessment. Southeast Asian J Trop Med Public Health. 2001;32:(2):229-35. 